\title{
Probing Gas-Phase Clustering Thermodynamics with Ion Mobility-Mass Spectrometry: Association Energies of Phenylalanine Ions with Gas-Phase Alcohols
}

Pearl Kwantwi-Barima, ${ }^{1}$ Christopher J. Hogan Jr., ${ }^{2}$ Brian H. Clowers ${ }^{1,}{ }^{*}$

${ }^{1}$ Department of Chemistry, Washington State University, Pullman, WA 99164

2 Department of Mechanical Engineering, University of Minnesota, Minneapolis, MN 55455

Corresponding Author:

Brian $\mathrm{H}$. Clowers

Washington State University

Department of Chemistry

E-mail: brian.clowers@wsu.edu

\section{Table of content:}

Equation S1

Equation S2

Equation S3

Equation S4

Equation S5

Figure S1

Table S1

Table S2

Table S3

Table S4

Table S5

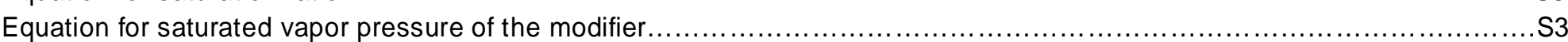

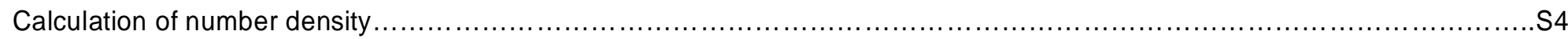

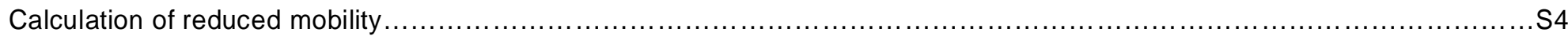

Equation for Gibbs free energy inferred from measurement based on a consistent reference state $\left(\Delta G_{1, r e f}\right) \ldots \ldots \ldots \ldots \ldots \ldots \ldots \ldots . \ldots \ldots$

Mass spectra of the target analyte ions at $120 \mu \mathrm{L} / \mathrm{hr}$ liquid feed flow rate of 2-propanol, 1-butanol and 2-pentanol ................S5 A summary of operating conditions for ion mobility spectrometer and mass spectrometer ....................................... 6

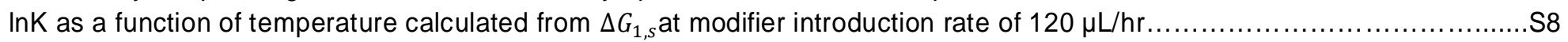

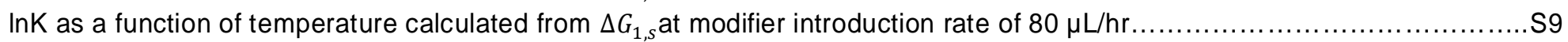

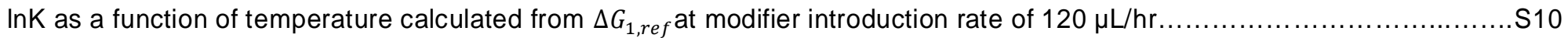

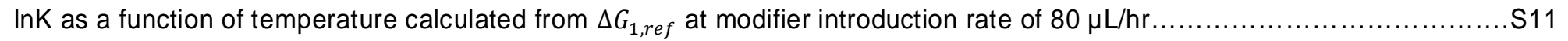


Table S6

Table S7

Table S8

Table S9
Gibbs free energy inferred at variable saturation state at any given measurement temperature $\left(\Delta G_{1, s}, 120 \mu \mathrm{L} / \mathrm{hr}\right)$

Gibbs free energy inferred at variable saturation state at any given measurement temperature $\left(\Delta G_{1, s}, 80 \mu \mathrm{L} / \mathrm{hr}\right) \ldots$

Gibbs free energy inferred at any given measurement temperature based on a consistent reference state $\left(\Delta G_{1, r e f}, 120 \mu \mathrm{L} / \mathrm{hr}\right) . . \mathrm{S} 14$

Gibbs free energy inferred at any given measurement temperature based on a consistent reference state $\left(\Delta G_{1, r e f}, 80 \mu \mathrm{L} / \mathrm{hr}\right) \ldots \mathrm{S} 15$ 
The saturation ratio $(S)$ is given by the equation:

$S=\frac{P}{P_{\text {sat }}(T)}$

where $P$ is the experimental vapor pressure of the modifier calculated from the concentration of the vapor modifier and the measurement temperature through the ideal gas law.

$P_{\text {sat }}(T)$ is the saturated vapor pressure of the modifier at the measurement temperature $\mathrm{T}$, calculated through Clausius-Clapeyron equation:

$P_{\text {sat }}(T)=P_{\text {ref }}\left(T_{\text {ref }}\right) \exp \left(-\frac{H_{\text {vap }}}{k}\left[\frac{1}{T}-\frac{1}{T_{\text {ref }}}\right]\right)$

where $P_{\text {ref }}\left(T_{r e f}\right)$ is the saturated vapor pressure of the modifier at a defined consistent reference temperature at $\mathrm{T}_{\text {ref }}(298 \mathrm{~K}), H_{v a p}$ is the enthalpy of vaporization for the vapor modifier, and $k$ is Boltzmann's constant. For example, with 2-propanol as a drift gas modifier, we demonstrate how saturation ratio (S) is calculated at $\mathrm{T}=453.15 \mathrm{~K}$, modifier flow rate $=120 \mu \mathrm{L} / \mathrm{hr}, \mathrm{N}_{2}$ gas flow rate $=120 \mathrm{~L} / \mathrm{hr}\left(3.33 \times 10^{-5} \mathrm{~m}^{3} / \mathrm{s}\right)$, liquid density $=785$ $\mathrm{kg} / \mathrm{m}^{3}$, molecular weight (2-propanol) $=60.1 \mathrm{~g} / \mathrm{mol}\left(9.983 \times 10^{-26} \mathrm{~kg} / \mathrm{molecule}\right), P_{\text {ref }}\left(T_{\text {ref }}\right)=5818.87 \mathrm{~Pa}$ (saturated vapor pressure of the modifier at $298 \mathrm{~K}), \mathrm{H}_{\mathrm{vap}}=45380 \mathrm{~J} / \mathrm{mol}, T_{\mathrm{ref}}=298.15 \mathrm{~K}$. The vapor number concentration of the modifier $\left(\mathrm{molecules} / \mathrm{m}^{3}\right)$ is calculated as the mass flow rate of the modifier $(\mathrm{kg} / \mathrm{s})$ divided by the $\mathrm{N}_{2}$ gas flow rate $\left(\mathrm{m}^{3} / \mathrm{s}\right)$ and the molecular weight of the modifier $(\mathrm{kg} / \mathrm{molecule})$. Subsequently, the experimental vapor pressure $P$ is the product of the vapor number concentration of the modifier, the measurement temperature and Boltzmann's constant. For the example conditions, the vapor pressure is $4.92 \times 10^{1} \mathrm{~Pa}$ and the vapor pressure at saturation is: $P_{\text {sat }}(T)=$

$P_{\text {ref }}\left(T_{\text {ref }}\right) \exp \left(-\frac{H_{\text {vap }}}{k}\left[\frac{1}{T}-\frac{1}{T_{\text {ref }}}\right]\right), P_{\text {sat }}(T)=3050474.80 \mathrm{~Pa}$

This yields the example saturation ratio $(\mathrm{S})=\frac{P}{P_{S a t}(T)}$

$=4.92 \times 10^{1} \mathrm{~Pa} / 3050474.76 \mathrm{~Pa}$

$=1.61 \times 10^{-5}$ 


\section{Reduced Mobility Calculation}

Number Density $=\left(2.68677 \times 10^{19}\right) *(273.15 \mathrm{~K} /$ Temperature $(\mathrm{K})) *($ Pressure $($ Torr $) / 760$ Torr $) \ldots$

$K_{0}($ reduced mobility $)=\left(1000 * L^{*}\right.$ Number density $) /\left(\left(t_{d}\right) *\left(2.68677 \times 10^{19}\right) * E\right)$

Where $\mathrm{L}=$ drift time length $(\mathrm{cm}), \mathrm{td}_{\mathrm{d}}=$ drift time $(\mathrm{ms}), \mathrm{E}=$ Electric field $(\mathrm{V} / \mathrm{cm})$

At any given temperature, the Gibbs free energy inferred from measurement based on a consistent reference state $\left(\Delta G_{1, r e f}\right)$ is linked to the Gibbs free energy at a variable saturation state via Equation S1.

$$
\Delta G_{1, r e f}=\Delta G_{1, S}-H_{v a p}\left(1-\frac{T}{T_{r e f}}\right)
$$



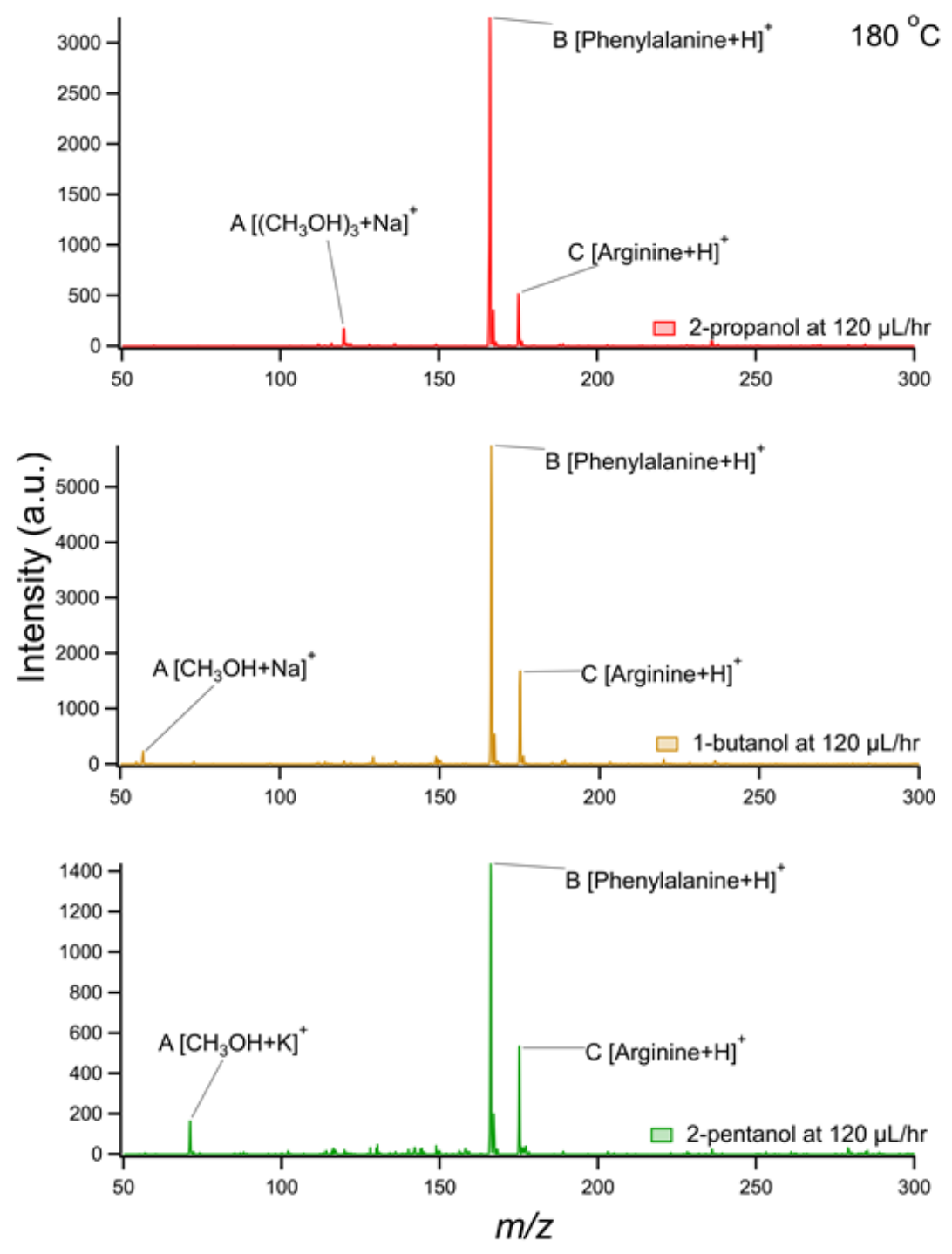

FIGURE S1. Mass spectrum of phenylalanine and arginine mixture ions when 2-propanol, 1-butanol and 2-pentanol were introduced into the system as drift gas modifiers at a flow rate of $120 \mu \mathrm{L} / \mathrm{hr}$. 
Table S1

\begin{tabular}{|c|c|c|}
\hline Parameter & Setting & stdev \\
\hline Drift tube length $(\mathrm{cm})$ & 10.56 & \pm 0.02 \\
\hline \multirow[t]{4}{*}{ Voltage at first ring (V) } & 9000 & \pm 0.02 \\
\hline & 8500 & \pm 0.01 \\
\hline & 8000 & \pm 0.01 \\
\hline & 7500 & \pm 0.02 \\
\hline \multirow[t]{4}{*}{ Voltage at the gate } & 4982.5 & \pm 0.02 \\
\hline & 4705.8 & \pm 0.01 \\
\hline & 4429 & \pm 0.01 \\
\hline & 4152.3 & \pm 0.02 \\
\hline Drift gas temperature/ drift tube temperature & $120-180$ & $\pm 0.2^{\circ} \mathrm{C}$ \\
\hline Pressure (Torr) & 690 & \pm 2 \\
\hline Mass spectrometer parameters & Setting & stdev \\
\hline Spray voltage (kV) & 0.00 & - \\
\hline Spray current $(\mu \mathrm{A})$ & 0.00 & - \\
\hline Capillary Temperature (oC) & $120-180$ & $\pm 0.2^{\circ} \mathrm{C}$ \\
\hline Capillary voltage (V) & 21.92 & \pm 0.5 \\
\hline Tube lens (V) & 54.98 & \pm 1.0 \\
\hline Multipole 00 offset (V) & -7.21 & \pm 0.3 \\
\hline Lens $0(\mathrm{~V})$ & -7.99 & \pm 0.3 \\
\hline
\end{tabular}




\begin{tabular}{|l|l|l|}
\hline Multipole 0 offset $(\mathrm{V})$ & -8.50 & \pm 0.3 \\
\hline Lens $1(\mathrm{~V})$ & -13.96 & \pm 0.3 \\
\hline Gate lens $(\mathrm{V})$ & -56.41 & \pm 0.6 \\
\hline Multipole 1 offset $(\mathrm{V})$ & -20.02 & \pm 0.4 \\
\hline Multipole RF $(\mathrm{Vp}-\mathrm{p})(\mathrm{V})$ & 0.46 & \pm 0.05 \\
\hline Front lens $(\mathrm{V})$ & 0.41 & \pm 0.02 \\
\hline Front section offset $(\mathrm{V})$ & 0.43 & \pm 0.02 \\
\hline Center section offset $(\mathrm{V})$ & 0.55 & \pm 0.03 \\
\hline Back section offset $(\mathrm{V})$ & 0.33 & \pm 0.02 \\
\hline Back lens $(\mathrm{V})$ & 0.06 & - \\
\hline
\end{tabular}

Table S1. A summary of the operating conditions for the ion mobility spectrometer and the mass spectrometer 
Table S2

\begin{tabular}{|c|c|c|c|c|c|c|}
\hline \multicolumn{7}{|c|}{ 2-propanol } \\
\hline Temperature/K & $\begin{array}{l}9000 \mathrm{~V} \\
\text { InK }\end{array}$ & $\begin{array}{l}8500 \mathrm{~V} \\
\text { InK }\end{array}$ & $\begin{array}{l}8000 \mathrm{~V} \\
\text { InK }\end{array}$ & $\begin{array}{l}7500 \mathrm{~V} \\
\operatorname{lnK}\end{array}$ & Average $\operatorname{lnK}$ & STDEV \\
\hline 453.15 & 13.9 & 13.9 & 14.0 & 14.0 & 13.9 & 0.1 \\
\hline 433.15 & 13.7 & 13.6 & 13.8 & 13.5 & 13.6 & 0.1 \\
\hline 413.15 & 13.4 & 13.3 & 13.2 & 13.3 & 13.3 & 0.1 \\
\hline 393.15 & 12.8 & 12.8 & 12.7 & 12.8 & 12.8 & 0.1 \\
\hline \multicolumn{7}{|c|}{ 1-butanol } \\
\hline 453.15 & 13.0 & 13.0 & 12.2 & 12.4 & 12.7 & 0.4 \\
\hline 433.15 & 12.6 & 12.5 & 12.5 & 12.5 & 12.5 & 0.1 \\
\hline 413.15 & 12.4 & 12.3 & 12.3 & 12.3 & 12.3 & 0.1 \\
\hline 393.15 & 11.9 & 11.8 & 11.9 & 12.0 & 11.9 & 0.1 \\
\hline \multicolumn{7}{|c|}{ 2-pentanol } \\
\hline 453.15 & 14.3 & 14.22 & 14.3 & 14.2 & 14.3 & 0.1 \\
\hline 433.15 & 13.9 & 14.13 & 14.2 & 14.3 & 14.2 & 0.2 \\
\hline 413.15 & 13.6 & 13.86 & 13.5 & 13.4 & 13.6 & 0.2 \\
\hline 393.15 & 12.9 & 13.3 & 12.8 & 13.5 & 13.1 & 0.3 \\
\hline
\end{tabular}

Table S2: Extracted inferred equilibrium constant (InKeq) as a function of inverse of temperature. The equilibrium constants (InKeq) were calculated from the inferred Gibbs free energy $\left(\Delta G_{1, S}\right)$ inferred using $\Omega_{1}$ from measurements at the modifier introduction rates of $120 \mu \mathrm{L} / \mathrm{hr}$ in Table S5.

Average InK for the phenylalanine ion was plotted as a function of inverse of temperature with the standard deviation as error bars in Figure 5. 
Table S3

\begin{tabular}{|c|c|c|c|c|c|c|}
\hline \multicolumn{7}{|c|}{ 2-propanol } \\
\hline Temperature/K & $\begin{array}{l}9000 \mathrm{~V} \\
\text { InK }\end{array}$ & $\begin{array}{l}8500 \mathrm{~V} \\
\text { InK }\end{array}$ & $\begin{array}{l}8000 \mathrm{~V} \\
\mathrm{InK}\end{array}$ & $\begin{array}{l}7500 \mathrm{~V} \\
\text { InK }\end{array}$ & Average $\ln \mathrm{K}$ & STDEV \\
\hline 453.15 & 13.9 & 14.2 & 14.1 & 14.3 & 14.1 & 0.2 \\
\hline 433.15 & 13.9 & 13.9 & 13.7 & 13.8 & 13.8 & 0.1 \\
\hline 413.15 & 13.5 & 13.6 & 13.5 & 13.3 & 13.5 & 0.1 \\
\hline 393.15 & 13.3 & 13.5 & 13.4 & 13.3 & 13.4 & 0.1 \\
\hline \multicolumn{7}{|c|}{ 1-butanol } \\
\hline 453.15 & 13.4 & 13.5 & 12.8 & 13.1 & 13.2 & 0.3 \\
\hline 433.15 & 12.9 & 12.7 & 12.7 & 12.7 & 12.8 & 0.1 \\
\hline 413.15 & 12.8 & 12.6 & 12.7 & 12.5 & 12.7 & 0.1 \\
\hline 393.15 & 12.4 & 12.3 & 12.1 & 12.3 & 12.3 & 0.2 \\
\hline \multicolumn{7}{|c|}{ 2-pentanol } \\
\hline 453.15 & 14.7 & 14.7 & 14.6 & 14.4 & 14.6 & 0.1 \\
\hline 433.15 & 14.3 & 14.6 & 14.5 & 14.5 & 14.5 & 0.2 \\
\hline 413.15 & 14.2 & 14.1 & 14.0 & 13.9 & 14.0 & 0.1 \\
\hline 393.15 & 13.2 & 13.3 & 13.3 & 13.7 & 13.3 & 0.2 \\
\hline
\end{tabular}

Table S3: Extracted inferred equilibrium constant (InKeq) as a function of inverse of temperature. The equilibrium constants (InKeq) were calculated from the inferred Gibbs free energy $\left(\Delta G_{1, s}\right)$ inferred using $\Omega_{1}$ from measurements at the modifier introduction rates of $80 \mu \mathrm{L} / \mathrm{hr}$ in Table S6 
Table S4

\begin{tabular}{|c|c|c|c|c|c|c|}
\hline \multicolumn{7}{|c|}{ 2-propanol } \\
\hline Temperature/K & $\begin{array}{l}9000 \mathrm{~V} \\
\text { InK }\end{array}$ & $\begin{array}{l}8500 \mathrm{~V} \\
\text { InK }\end{array}$ & $\begin{array}{l}8000 \mathrm{~V} \\
\operatorname{lnK}\end{array}$ & $\begin{array}{l}7500 \mathrm{~V} \\
\text { InK }\end{array}$ & Average $\ln K$ & STDEV \\
\hline 453.15 & 7.66 & 7.64 & 7.71 & 7.79 & 7.70 & 0.06 \\
\hline 433.15 & 8.04 & 7.89 & 8.06 & 7.82 & 7.95 & 0.11 \\
\hline 413.15 & 8.35 & 8.26 & 8.16 & 8.26 & 8.26 & 0.08 \\
\hline 393.15 & 8.40 & 8.36 & 8.32 & 8.43 & 8.38 & 0.05 \\
\hline \multicolumn{7}{|c|}{ 1-butanol } \\
\hline 453.15 & 5.84 & 5.84 & 5.00 & 5.15 & 5.46 & 0.33 \\
\hline 433.15 & 6.01 & 5.97 & 5.92 & 5.90 & 5.95 & 0.05 \\
\hline 413.15 & 6.57 & 6.45 & 6.41 & 6.43 & 6.46 & 0.07 \\
\hline 393.15 & 6.80 & 6.70 & 6.78 & 6.93 & 6.80 & 0.11 \\
\hline \multicolumn{7}{|c|}{ 2-pentanol } \\
\hline 453.15 & 6.19 & 6.11 & 6.21 & 6.11 & 6.15 & 0.05 \\
\hline 433.15 & 6.57 & 6.73 & 6.85 & 6.91 & 6.76 & 0.15 \\
\hline 413.15 & 7.04 & 7.25 & 6.93 & 6.82 & 7.01 & 0.18 \\
\hline 393.15 & 7.23 & 7.58 & 7.07 & 7.75 & 7.41 & 0.31 \\
\hline
\end{tabular}

Table S4: Extracted inferred equilibrium constant (InK $\mathrm{K}_{\mathrm{eq}}$ ) as a function of inverse of temperature. The inferred equilibrium constants (InK $\mathrm{K}_{\mathrm{eq}}$ ) were calculated from Gibbs free energy $\left(\Delta G_{1, \text { ref }}\right)$ in Table S7 inferred using $\Omega_{1}$ from measurements at the modifier introduction rate of $120 \mu \mathrm{L} / \mathrm{hr}$.

Average InK for the phenylalanine ion was plotted as a function of inverse of temperature with the standard deviation as error bars in Figure 6 
Table S5

\begin{tabular}{|c|c|c|c|c|c|c|}
\hline \multicolumn{7}{|c|}{ 2-propanol } \\
\hline Temperature/K & $\begin{array}{l}9000 \mathrm{~V} \\
\text { InK }\end{array}$ & $\begin{array}{l}8500 \mathrm{~V} \\
\operatorname{lnK}\end{array}$ & $\begin{array}{l}8000 \mathrm{~V} \\
\text { InK }\end{array}$ & $\begin{array}{l}7500 \mathrm{~V} \\
\operatorname{lnK}\end{array}$ & Average InK & STDEV \\
\hline 453.15 & 7.68 & 7.99 & 7.89 & 8.03 & 7.90 & 0.12 \\
\hline 433.15 & 8.28 & 8.28 & 8.03 & 8.07 & 8.16 & 0.13 \\
\hline 413.15 & 8.42 & 8.53 & 8.45 & 8.26 & 8.41 & 0.11 \\
\hline 393.15 & 8.87 & 9.06 & 9.02 & 8.94 & 8.97 & 0.09 \\
\hline \multicolumn{7}{|c|}{ 1-butanol } \\
\hline 453.15 & 6.17 & 6.28 & 5.64 & 5.86 & 5.99 & 0.29 \\
\hline 433.15 & 6.30 & 6.16 & 6.15 & 6.12 & 6.18 & 0.08 \\
\hline 413.15 & 6.92 & 6.75 & 6.85 & 6.66 & 6.79 & 0.11 \\
\hline 393.15 & 7.28 & 7.22 & 6.96 & 7.19 & 7.16 & 0.14 \\
\hline \multicolumn{7}{|c|}{ 2-pentanol } \\
\hline 453.15 & 6.55 & 6.56 & 6.49 & 6.29 & 6.47 & 0.13 \\
\hline 433.15 & 6.86 & 7.20 & 7.07 & 7.13 & 7.07 & 0.15 \\
\hline 413.15 & 7.54 & 7.48 & 7.37 & 7.27 & 7.42 & 0.12 \\
\hline 393.15 & 7.44 & 7.58 & 7.53 & 7.92 & 7.62 & 0.21 \\
\hline
\end{tabular}

Table S5: Extracted inferred equilibrium constant (InKeq) as a function of inverse of temperature. The inferred equilibrium constants (InKeq) were calculated from Gibbs free energy $\left(\Delta G_{1, \text { ref }}\right)$ in Table $\mathrm{S} 8$ inferred using $\Omega_{1}$ from measurements at the modifier introduction rates of $80 \mu \mathrm{L} / \mathrm{hr}$. 
Table S6

\begin{tabular}{|c|c|c|c|c|c|c|}
\hline \multicolumn{7}{|c|}{ 2-propanol } \\
\hline Temperature/K & $\begin{array}{l}9000 \mathrm{~V} \\
\Delta \square_{1, \square}(\mathrm{kcal} / \mathrm{mol})\end{array}$ & $\begin{array}{l}8500 \mathrm{~V} \\
\Delta \square_{1, \square}(\mathrm{kcal} / \mathrm{mol})\end{array}$ & $\begin{array}{l}8000 \mathrm{~V} \\
\Delta \square_{1, \square}(\mathrm{kcal} / \mathrm{mol})\end{array}$ & $\begin{array}{l}7500 \mathrm{KV} \\
\Delta \square_{1, \square}(\mathrm{kcal} / \mathrm{mol})\end{array}$ & $\begin{array}{l}\text { Average } \Delta \square 1, \square \\
\text { (kcal/mol) }\end{array}$ & STDEV \\
\hline 453.15 & -12.55 & -12.53 & -12.59 & -12.66 & -12.6 & 0.1 \\
\hline 433.15 & -11.84 & -11.71 & -11.85 & -11.65 & -11.8 & 0.1 \\
\hline 413.15 & -11.05 & -10.98 & -10.89 & -10.98 & -10.9 & 0.1 \\
\hline 393.15 & -10.02 & -10.00 & -9.97 & -10.05 & -10.0 & 0.1 \\
\hline \multicolumn{7}{|c|}{ 1-butanol } \\
\hline 453.15 & -11.77 & -11.77 & -11.01 & -11.15 & -11.4 & 0.4 \\
\hline 433.15 & -10.85 & -10.81 & -10.77 & -10.75 & -10.8 & 0.1 \\
\hline 413.15 & -10.22 & -10.13 & -10.10 & -10.11 & -10.2 & 0.1 \\
\hline 393.15 & -9.31 & -9.23 & -9.29 & -9.41 & -9.31 & 0.08 \\
\hline \multicolumn{7}{|c|}{ 2-pentanol } \\
\hline 453.15 & -12.90 & -12.83 & -12.92 & -12.83 & -12.9 & 0.1 \\
\hline 433.15 & -12.04 & -12.18 & -12.28 & -12.33 & -12.2 & 0.1 \\
\hline 413.15 & -11.22 & -11.39 & -11.13 & -11.04 & -11.2 & 0.2 \\
\hline 393.15 & -10.14 & -10.42 & -10.02 & -10.55 & -10.3 & 0.2 \\
\hline
\end{tabular}

Table S6: Gibbs free energy inferred from the experiment at any given measurement temperature based on a variable saturation state $\left(\Delta G_{1, s}\right)$ inferred using $\Omega_{1}$ from measurements at the modifier introduction rates of $120 \mu \mathrm{L} / \mathrm{hr}$. Average Gibbs free energy change for the phenylalanine target analyte ion is recorded in Table 3.

Table S7 


\begin{tabular}{|c|c|c|c|c|c|c|}
\hline \multicolumn{7}{|c|}{ 2-propanol } \\
\hline Temperature/K & $\begin{array}{l}9000 \mathrm{~V} \\
\Delta \square_{1, \square}(\mathrm{kcal} / \mathrm{mol})\end{array}$ & $\begin{array}{l}8500 \mathrm{~V} \\
\Delta \square_{1, \square}(\mathrm{kcal} / \mathrm{mol})\end{array}$ & $\begin{array}{l}8000 \mathrm{~V} \\
\Delta \square_{1, \square}(\mathrm{kcal} / \mathrm{mol})\end{array}$ & $\begin{array}{l}7500 \mathrm{KV} \\
\Delta \square_{1, \square}(\mathrm{kcal} / \mathrm{mol})\end{array}$ & $\begin{array}{l}\text { Average } \Delta \square 1, \square \\
\text { (kcal/mol) }\end{array}$ & STDEV \\
\hline 453.15 & -12.6 & -12.8 & -12.7 & -12.9 & -12.8 & 0.1 \\
\hline 433.15 & -12.1 & -12.1 & -11.8 & -11.9 & -11.9 & 0.2 \\
\hline 413.15 & -11.1 & -11.2 & -11.1 & -10.9 & -11.1 & 0.1 \\
\hline 393.15 & -10.4 & -10.5 & -10.5 & -10.4 & -10.5 & 0.1 \\
\hline \multicolumn{7}{|c|}{ 1-butanol } \\
\hline 453.15 & -12.1 & -12.2 & -11.6 & -11.8 & -11.9 & 0.3 \\
\hline 433.15 & -11.1 & -11.0 & -10.9 & -10.9 & -11.0 & 0.1 \\
\hline 413.15 & -10.5 & -10.4 & -10.4 & -10.3 & -10.4 & 0.1 \\
\hline 393.15 & $\mid-9.68$ & -9.63 & -9.43 & $\mid-9.61$ & -9.59 & 0.11 \\
\hline \multicolumn{7}{|c|}{ 2-pentanol } \\
\hline 453.15 & -13.2 & -13.2 & -13.2 & -13.0 & -13.1 & 0.1 \\
\hline 433.15 & -12.3 & -12.6 & -12.5 & -12.5 & -12.5 & 0.1 \\
\hline 413.15 & -11.6 & -11.6 & -11.5 & -11.4 & -11.5 & 0.1 \\
\hline 393.15 & -10.3 & -10.4 & -10.4 & -10.4 & -10.4 & 0.2 \\
\hline
\end{tabular}

Table S7: Gibbs free energy inferred from the experiment at any given measurement temperature based on a variable saturation state $\left(\Delta G_{1, s}\right)$ inferred using $\Omega_{1}$ from measurements at the modifier introduction rates of $80 \mu \mathrm{L} / \mathrm{hr}$. 
Table S8

\begin{tabular}{|c|c|c|c|c|c|c|}
\hline \multicolumn{7}{|c|}{ 2-propanol } \\
\hline Temperature/K & $\begin{array}{l}9000 \mathrm{~V} \\
\Delta \square_{1, \square \square \square}(\mathrm{kcal} / \mathrm{mol})\end{array}$ & $\begin{array}{l}8500 \mathrm{~V} \\
\Delta \square_{1, \square \square \square}(\mathrm{kcal} / \mathrm{mol})\end{array}$ & $\begin{array}{l}8000 \mathrm{~V} \\
\Delta \square_{1, \square \square \square}\end{array}(\mathrm{kcal} / \mathrm{mol})$ & $\begin{array}{l}7500 \mathrm{~V} \\
\Delta \square_{1, \square \square \square}(\mathrm{kcal} / \mathrm{mol})\end{array}$ & 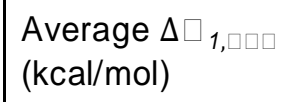 & STDEV \\
\hline 453.15 & -6.91 & -6.89 & -6.95 & -7.02 & -6.94 & 0.06 \\
\hline 433.15 & -6.93 & -6.80 & -6.94 & -6.74 & -6.85 & 0.11 \\
\hline 413.15 & -6.87 & -6.79 & -6.71 & -6.79 & -6.79 & 0.06 \\
\hline 393.15 & -6.57 & -6.54 & -6.51 & -6.60 & -6.55 & 0.04 \\
\hline \multicolumn{7}{|c|}{ 1-butanol } \\
\hline 453.15 & -5.27 & -5.26 & -4.51 & -4.65 & -4.92 & 0.40 \\
\hline 433.15 & -5.18 & -5.15 & -5.10 & -5.09 & -5.13 & 0.04 \\
\hline 413.15 & -5.40 & -5.30 & -5.27 & -5.29 & -5.32 & 0.06 \\
\hline 393.15 & -5.32 & -5.24 & -5.30 & -5.42 & -5.32 & 0.08 \\
\hline \multicolumn{7}{|c|}{ 2-pentanol } \\
\hline 453.15 & -5.58 & -5.51 & -5.60 & -5.51 & -5.55 & 0.05 \\
\hline 433.15 & -5.66 & -5.80 & -5.90 & -5.96 & -5.83 & 0.13 \\
\hline 413.15 & -5.79 & -5.96 & -5.70 & -5.61 & -5.76 & 0.15 \\
\hline 393.15 & -5.66 & -5.93 & -5.53 & -6.07 & -5.80 & 0.25 \\
\hline
\end{tabular}

Table S8: Gibbs free energy inferred at a consistent reference state $\left(\Delta G_{1, r e f}\right)$ through equation S1 at any given measurement temperature inferred using $\Omega_{1}$ from measurements at the modifier introduction rates of $120 \mu \mathrm{L} / \mathrm{hr}$. Average Gibbs free energy change for the phenylalanine target analyte ion is recorded in Table 4. 
Table S9

\begin{tabular}{|c|c|c|c|c|c|c|}
\hline \multicolumn{7}{|c|}{ 2-propanol } \\
\hline Temperature/K & $\begin{array}{l}9000 \mathrm{~V} \\
\Delta \square_{1, \square \square \square}(\mathrm{kcal} / \mathrm{mol})\end{array}$ & $\begin{array}{l}8500 \mathrm{~V} \\
\Delta \square_{1, \square \square \square}(\mathrm{kcal} / \mathrm{mol})\end{array}$ & $\begin{array}{l}8000 \mathrm{~V} \\
\Delta \square_{1, \square \square \square}(\mathrm{kcal} / \mathrm{mol})\end{array}$ & $\begin{array}{l}7500 \mathrm{~V} \\
\Delta \square_{1, \square \square \square}(\mathrm{kcal} / \mathrm{mol})\end{array}$ & 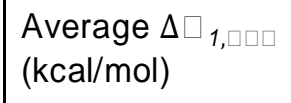 & STDEV \\
\hline 453.15 & -6.92 & --7.21 & -7.12 & -7.24 & -7.12 & 0.14 \\
\hline 433.15 & -7.14 & -7.13 & -6.92 & -6.95 & -7.04 & 0.12 \\
\hline 413.15 & -6.93 & -7.01 & -6.94 & -6.79 & -6.92 & 0.09 \\
\hline 393.15 & -6.94 & -7.09 & -7.06 & -6.99 & -7.02 & 0.07 \\
\hline \multicolumn{7}{|c|}{ 1-butanol } \\
\hline 453.15 & -5.57 & -5.66 & -5.09 & -5.28 & -5.40 & 0.26 \\
\hline 433.15 & -5.43 & -5.31 & -5.31 & -5.27 & -5.33 & 0.07 \\
\hline 413.15 & 5.69 & -5.55 & -5.63 & -5.47 & -5.58 & 0.09 \\
\hline 393.15 & -5.69 & -5.65 & -5.45 & -5.63 & -5.60 & 0.11 \\
\hline \multicolumn{7}{|c|}{ 2-pentanol } \\
\hline 453.15 & -5.91 & -5.92 & -5.85 & -5.67 & -5.84 & 0.12 \\
\hline 433.15 & -5.91 & -6.21 & -6.10 & -6.15 & -6.09 & 0.13 \\
\hline 413.15 & -6.20 & -6.15 & -6.06 & -5.98 & -6.10 & 0.11 \\
\hline 393.15 & -5.82 & -5.93 & -5.89 & -6.20 & -5.96 & 0.17 \\
\hline
\end{tabular}

Table S9: Gibbs free energy inferred at a consistent reference state $\left(\Delta G_{1, r e f}\right)$ through equation S1 at any given measurement temperature, inferred using $\Omega_{1}$ from measurements at the modifier introduction rates of $80 \mu \mathrm{L} / \mathrm{hr}$. 